\title{
Globalisation, terrain et théorie : l'anthropologie retraversée
}

François Laplantine et Francine Saillant

\section{(2) OpenEdition \\ 1 Journals}

Édition électronique

URL : https://journals.openedition.org/pa/1848

DOI : 10.4000/pa.1848

ISSN : 2273-0362

Éditeur

Université Lumière Lyon 2

Édition imprimée

Date de publication : 1 janvier 2005

Pagination : 10-17

ISBN : 1634-7706

ISSN : 1634-7706

Référence électronique

François Laplantine et Francine Saillant, « Globalisation, terrain et théorie : I'anthropologie retraversée », Parcours anthropologiques [En ligne], 5 | 2005, mis en ligne le 06 juillet 2021, consulté le 21 juillet 2021. URL : http://journals.openedition.org/pa/1848; DOI : https://doi.org/10.4000/pa.1848 


\section{GLOBALISATION, TERRAIN ET THÉORIE :}

\section{L'ANTHROPOLOGIE RETRAVERSÉE}

\section{Globalisation et décloisonnement des} terrains

Les questions posées par ce que l'on appelle en Amérique latine et en Amérique du Nord la globalisation et en Europe la mondialisation constituent un défi pour l'anthropologie, laquelle est d'abord ethnographie. La globalisation est un processus immense, macroscopique, alors que la démarche ethnographique est une démarche micrologique. Autrement dit, nous ne pouvons, en ethnographie, étudier des processus macroscopiques qu'en montrant comment ils s'inscrivent dans de minuscules interactions. Ce n'est qu'à partir du micro que les ethnographes peuvent aller vers le macro. Ce n'est qu'à travers le local qu'ils peuvent analyser le global, en évitant deux tentations : l'une, l'orgueil de la généralisation hâtive du concept, la prétention à l'universalisme abstrait; l'autre, l'enfermement dans l'histoire régionale.

Jacques Revel nous propose une notion qui nous semble particulièrement opératoire : la "variation d'échelle d'observation » ou "variation de la focale" $"$ qui consiste tantôt à nous approcher, tantôt à nous éloigner. L'expérience de la "variation d'échelle d'observation" est une expérience physique que nous réalisons tant dans l'ethnographie que dans le cinéma qui sont pour ainsi dire condamnés à la singularité concrète d'un point de vue alors que ce qui leur est interdit est le point de vue unique d'un spectateur omniprésent et omnipotent. Le recours au cinéma constitue pour les anthropologues une incitation à une extrême précision. Il pose d'emblée, comme l'ethnographie, la question de la double relation à un temps particulier et à un espace singulier. Dans l'expérience du terrain, que nous définirons comme une expérience du partage du sensible, nous effectuons des observations (ce qui pose la question du rapport à l'espace et notamment aux images) et nous menons des entretiens (nous sommes ainsi confrontés aux trois familles de sons : la voix, les bruits et la musique), au temps et à l'histoire. Or c'est ce temps qui dans le processus de mondialisation s'accélère alors que l'espace, lui, paraît rétrécir.

Nous partirons d'un film qui nous montre un espace isolé ainsi qu'une temporalité arrêtée, un espace-temps pour ainsi dire coupé du reste du monde et nous verrons que, même dans cet exemple à contrario, les processus en question ont bien lieu. Il s'agit d'Il Dono (2003) de Michelangelo Framartini qui se passe dans un village de Calabre où les destins d'un vieil homme et d'une jeune fille vont se croiser.

Le film commence dans une ferme où le premier vient de faire appel à des fossoyeurs de fortune pour enterrer son chien. L'un d'eux a oublié sur la table de la maison une photo pornographique. Framartini filme l'existence quotidienne et plus particulièrement le vieillissement d'une population isolée qui continue à vivre dans le temps de l'ancienne religion populaire. Ce vieillissement est montré à travers la rouille des carcasses de voitures abandonnées ainsi que les rides de la majorité des habitants, lesquels ne sont pas des acteurs professionnels et interprètent leurs propres rôles.

Dans ce film d'une extrême retenue narrative, sans dialogue, mais construit dans un travail minutieux de la bande son, il existe néanmoins une relation entre le local et le mondial. Cette relation s'effectue, comme c'est le propre du cinéma et de l'ethnographie, non à partir des idées mais à travers des sons et des images ou plus précisément entre des sons et des images. C'est le son strident du téléphone portable que le vieil homme a toujours à proximité de lui qui relie le local au global, l'ici à l'ailleurs ainsi que le passé au présent. Le téléphone portable relie les temporalités de l'ancien et du contemporain. Il fait franchir deux siècles. Le micro-local rencontre également le global à travers la photo pornographique ainsi qu'un scooter que le vieil homme offre (d'où le don, titre du film) à la jeune fille.

C'est dans la sérénité de chaque plan, qui sont pour la plupart de longs plans fixes, dans la légère angoisse du passage d'un plan à un autre ainsi que dans le travail de la bande son que nous percevons les relations entre le local et le global, le passé et le présent, le passage. Ce que nous percevons est la complexité de temporalités qui n'avancent pas au même rythme pour le vieillard et pour la jeune fille.

Il Dono a le mérite de mettre en scène, de manière sobre et précise, la communication, si minime soit-elle, des espaces produisant des temporalités différenciées. Alors que Mondovino (2003) de Nossiter dont il a été question dans le précédent numéro de Parcours Anthropologiques ou Le cauchemar de Darwin (2003) de Sauper dont nous parlerons plus loin montrent, quant à eux, l'accélération du temps mondialisé qui est aussi celui de la mercantilisation.

Le mérite de ces films est de mettre en question ce qui a constitué le piège de l'anthropologie classique dans sa tendance à privilégier le local contre le global, alors qu'ils vont ensemble. Ils éclairent des processus qui nous incitent à réinterroger la notion même de culture comme la chérissaient tant les premiers ethnologues, dotée d'une cohérence et d'une cohésion interne et 
devant peu à ce qui, du « dehors ", pouvait venir l'altérer.

L'espace référentiel de l'ethnologie classique a bien été effectivement le local, le territoire lié à la notion de terrain. Les argonautes du Pacifique occidental (1922) de Malinowski ou L'homme d'Aran (1934) de Flaherty décrivent des îles coupées du monde, mieux des îles qui sont un monde, enfermant le chercheur, et avec lui le lecteur ou le spectateur, dans ce que Jean-Louis Amselle a appelé un "huis-clos» anthropologique.

L'ethnologie classique nous apparaît rétrospectivement comme une ethnologie coincée (dans des habitudes de délimitation) et confinée (dans des espaces fermés). Elle postule en quelque sorte qu'au début il y avait des cultures distinctes les unes des autres. Ce n'est seulement que par la suite, que les flux devenus plus intensifs, auraient entraîné des transformations. Autrement ce qui était premier ontologiquement et chronologiquement aurait produit de manière contingente du dérivé et de l'hybride.

Ce qui est tenu pour des ensembles clos est en fait une certaine manière de rendre compte de ces derniers en les essentialisant et en les déshistoricisant et ce qui est appelé "hybridation" ou "métissage " ne renvoie souvent qu'à la tentative assez désespérée du chercheur déconcerté par ce qui échappe aux catégories dualistes dans lesquelles il a été formé. Ce qu'il convient d'interroger dès lors, ce sont moins les cultures existantes (lesquelles n'ont jamais été pures, compactes et homogènes) que les instruments épistémologiques dont on se dote pour les étudier qui ont conduit à un enfermement spatial (et corrélativement à une spatialisation de la pensée) et ont produit une espèce d'ontologie différentielle.

Dans cette approche, tout se passe comme si nous n'avions pas d'autre choix épistémologique que l'universalité de la structure ou la particularité de la culture et pas d'autre choix politique que l'assimilation progressive ou la fragmentation différentialiste. Ces dualismes sémiologiques de l'identité absolue et pour ainsi dire inhérente et de l'altérité totale (à l'intérieur du groupe "nous" serions tous pareils alors qu'à l'extérieur, "ils » seraient différents) doivent être remis en question. Ces catégories oppositionnelles de l'identité et de l'altérité, du dedans et du dehors sont des notions classificatoires essentialisantes qui n'ont de pertinence que dans la logique inéquivoque et stabilisante qui est celle du signe.

Or il n'est pas sûr, que, confrontés aux questions posées par les processus contemporains de mondialisation, nous ayons véritablement changé de paradigme. Nous avons encore tendance à entretenir la fiction d'une pureté de l'endogène (le territoire) confronté à une violence qui ne serait qu'exogène, autrement dit à une conception d'imposition d'un modèle homogène à des ensembles hétérogènes. Il nous paraît pour le moins insuffisant de placer le préfixe inter devant tous les mots qui antérieurement connotaient des ensembles clos et statiques, c'est-à-dire devant des catégories censées être pures et homogènes.
Dans la fameuse "interculturalité ", la perspective du chercheur peut rester identique à ce qu'elle était. Elle peut rester occidentalocentrée. Cette perspective est celle d'un sujet durkheimien eurocentré c'est-à-dire blanc, masculin, hétérosexuel, compact, constant, cohérent, impassible, adéquat à lui-même. La rationalité anthropologique, tout en paraissant ouverte à la pluralité, continue à être pensée dans des termes monoculturels.

$\mathrm{Si}$ les choses commencent à changer en anthropologie, c'est à partir des périphéries, des sociétés diasporiques dans lesquelles se forment des cultures bilingues, trilingues, polymorphes, polyphoniques caractérisées notamment, comme au Brésil ou dans les Caraïbes, par des musiques antiphoniques qui ne sont plus composées à partir d'un principe harmonique d'équilibre et de centralité. La pensée anthropologique n'est plus seulement confrontée aujourd'hui à de l'autonome à de l'autochtone, à des racines, mais à ce que Gilles Deleuze appelle des "rhizomes ", Jean-Lou Amselle des "branchements " et Florence Dupont des processus d' « altérité incluse ».

La diaspora peut entretenir ou distendre le lien au pays (réel ou imaginaire) d'origine. Elle conduit les populations à tisser d'autres liens que ceux de la soumission à la communauté ou de l'intégration absolue. Et elle appelle l'anthropologue a être attentif à des cultures étrangères à l'enfermement dans des catégories ethniques et territoriales c'est-à-dire à des cultures itératives, ambulantes, ambivalentes, dissidentes, dissonantes et désorientées. Ainsi aujourd'hui un terrain ethnographique n'est plus lié nécessairement à un territoire. On s'en aperçoit particulièrement avec l'ouvrage de Laëtitia Atlani-Duault ${ }^{2}$ L'anthropologue se trouve confronté à des groupes déterritorialisés. Haïti, Cuba, Porto Rico existent aussi aujourd'hui à New-York, à Montréal et à Londres. Le Portugal existe aussi et même peut être surtout (et ce phénomène n'a rien de nouveau) à l'extérieur de lui-même : dans les Iles du CapVert, au Brésil, à Boston. Le véritable objet de l'anthropologie a, en fait, toujours été ce que nous appelons aujourd'hui globalisation ou mondialisation. Seule la conception du terrain comme huis-clos a pu conduire à penser que nos objets s'étaient depuis quelques années radicalement transformés.

\section{Mouvements des groupes minorisés et} anthropologies périphériques

Les anthropologies périphériques constituent à leur façon une autre proposition de variation d'échelle pour l'anthropologie. Elles se trouvent largement stimulées par certaines conditions de la globalisation dont la circulation des personnes et des intellectuels, celle des idées à travers les médias électroniques et enfin par la constitution de réseaux que sont les mouvements sociaux et les diasporas. Mais cette fois-ci, la variation d'échelle vient probablement de la perspective et de la position de celui qui parle, et surtout du fait de son appartenance, ou encore de son identification forte à l'un des groupes initialement étudiés par l'anthropologie, groupes 
représentant autant de flexions de figures de l'autre que la discipline a longuement réifiées et englobées sous le vocable de culture. Bien sûr, les sciences sociales et, encore plus parmi elles, l'anthropologie, composent avec " un objet " qui est un sujet de langage situé dans un monde social singulier. En anthropologie, ce sujet de langage a trop longtemps été placé à distance et en quelque sorte figé dans cette position de "représentant de l'Autre ", venant conforter et confirmer les représentations d'un Soi extérieur, porteur de rationalité et de vérité universelle. Le couple de l'identité et de l'altérité en anthropologie n'est-il pas pourtant l'héritage des rapports "Occident » et "Orient », "Nord " et «Sud », «Ouest» et «Est », reproduisant le "nous» et le «eux»du grand partage colonial qui a refoulé longuement tout projet d'anthropologie symétrique, au sens que lui donne Bruno Latour. Transposée dans la relation aux groupes et individus rencontrés par les anthropologues, la relation nous-eux héritée du partage colonial a enfermé la parole de l'autre dans celle d'éternel informateur, donc de sujet sans savoir, ou plutôt de sujet non instruit de son savoir. L'anthropologue s'est fait le plus souvent le décrypteur et l'archéologue de cultures et d'altérités que le savoir anthropologique expert aurait fait apparaître. L'autre fut alors celui par qui l'exotique arrive, comme jadis les Relations de voyage, Marco Polo et Jacques Cartier, les Jésuites et le Capitaine Cook, informaient des "mœurs et coutumes " des barbares et des sauvages. Les anthropologues ont placé la parole des informateurs dans une sorte de cabinet de curiosités des variations culturelles et ce, jusqu'à l'avènement du postmodernisme en anthropologie, qui ébranla bien des certitudes. Il fallu, en effet, que l'anthropologie puisse prendre conscience des modalités de son propre discours, de ses techniques de représentations des altérités, de ses formes d'écriture, qu'elle remette en question l'autorité ethnographique, comme le firent les Clifford, Geertz, etc., pour qu'un changement d'attitude et de regard puisse être rendu possible. Au cours de la même période, soit les années 1980, les anthropologies périphériques se sont développées de façon exponentielle, et celui qui fut l'Autre resurgit au sein même du discours anthropologique, cette fois-ci en tant que sujet de savoir, en développant, pour la première fois dans l'histoire de la discipline, les conditions d'une anthropologie symétrique et postcoloniale. Le croisement des anthropologies postmoderniste, symétrique et postcoloniale, est de notre point de vue le résultat de plusieurs processus au sein de la discipline. Il favorise un changement d'échelle toujours en train de se faire. Les anthropologies périphériques ne relèvent pas d'une simple inversion du rapport entre l'anthropologue et l'informateur, renversement de type hegelien, mais d'un brouillage radical des références pour toute l'anthropologie. Ce brouillage des références n'est pas tant une crise de la scientificité de l'anthropologie, ou encore une lubie anglosaxonne (parce que ce mouvement est venu surtout des pays anglo-saxons), qu'une occasion de re-définir la discipline en tenant compte de «celui par qui le savoir arrive». Nous voulons désigner ici le savoir de ceux qui, devenus instruits par et au sein même de l'anthropologie, et historiquement constitués en tant que sujets de l'altérité, deviennent maintenant les critiques les plus exigeants de la discipline, et élaborent un savoir anthropologique original. Ne pas tenir compte du savoir produit dans ce mouvement de renversement, qui est donc aussi un changement d'échelle et de perspective, signifierait enfermer l'anthropologie dans le grand partage et l'héritage colonial.

C'est ainsi que depuis maintenant plusieurs années, les femmes, les Noirs, les homosexuels, les Indiens, les Orientaux, pour ne nommer que ces derniers, ont permis l'avènement des anthropologies périphériques, c'est-à-dire des anthropologies aux prétentions non universalistes, aux formes localisées et plurielles, donnant enfin une voie à ceux qui "donnaient" le savoir aux anthropologues sans jamais maîtriser la direction et les formes de ce savoir, et sans pouvoir contester le savoir anthropologique produit à leur sujet.

Il faut rappeler que le premier brouillage à survenir au sein de la discipline, avant même qu'il ne soit question des études poscoloniales, a pour origine la rencontre du féminisme et de l'anthropologie. Les anthropologues classiques ont eu souvent des hommes et des chefs comme informateurs. Sur le plan épistémologique, cela signifiait de rendre compte du « monde de l'autre " à partir d'une seule position de genre, à partir d'une expérience située du monde, et d'exclure en tant que source de savoir et d'autorité sur la connaissance privilégiée du milieu étudié, sans nécessairement qu'il y ait là une intention, la moitié du monde. Toute l'expérience féminine se trouvait alors transmise, traduite ou gommée par des informateurs qui s'en faisaient les observateurs, les révélateurs et les témoins, informateurs masculins. Le féminisme en anthropologie a entraîné cette prise de conscience d'une science trop longtemps androcentrique et à partir d'elle, le début d'une écriture laissant apparaître "la voie des femmes ", que l'on soit d'ailleurs féministe ou non. Cela signifiait aussi d'accepter qu'il puisse y avoir plusieurs voix au sein même du discours anthropologique et de développer des formes différentes d'écriture, dites à plusieurs voix ou polyphoniques, mettant en scène l'anthropologue, mais aussi les subjectivités de plusieurs groupes et catégories identifiées au genre féminin. Le genre féminin ne pouvait plus être abordé au sein d'une même culture comme homogène et univocal; il se devait d'être abordé comme hétérogène et polyvolcal. Dans ce mouvement, non seulement des femmes prêtaient-elles leur voix à l'oreille de l'anthropologue, mais des anthropologues femmes se sont également introduites dans le discours anthropologique en tant que sujet engagé, singulier, dialogique, réflexif et gendered, ce qui voulait dire agir à contrario d'un sujet supposé neutre et universel, et d'une posture le plus souvent "masculin". Certains ont vu en ceci le danger d'un certain essentialisme au féminin, mais la question se devait d'être posée. Cela a même donné lieu à la relecture de terrains effectués par les maîtres de 
l'anthropologie, d'une part, et à la redécouverte des chemins empruntés par des femmes anthropologues, certaines très célèbres, c'est le cas de Margaret Mead, et d'autres moins célèbres, si l'on songe à Ruth Landes ${ }^{3}$.

Quoiqu'à l'extérieur de la France il soit vraiment hérétique de considérer l'apport du féminisme en anthropologie comme une recherche "à la périphérie", si l'on considère l'immensité du travail accompli et l'importance de ce courant dans tous les programmes d'anthropologie des universités dans le monde, il semble tout de même acceptable de nommer périphérique ce qui se constitue autrement que par et dans le centre et ne s'élabore point comme savoir désiré universel. L'universalisme en anthropologie a pris la place du commun, hors de l'émergence des subjectivités singulières. La connaissance issue des anthropologies périphériques traite autrement que par l'universalisme cette question du commun.

En sus de cette rencontre du féminisme et de l'anthropologie, une série d'anthropologies périphériques allaient ensuite se constituer à travers les black studies, les gay and lesbian studies, les postcolonial studies, influencées à la fois par le féminisme mais aussi par le postmodernisme et surtout par les cultural studies. Ainsi, autour des cultural studies ${ }^{4}$, toute une réflexion critique s'est élaborée, s'appuyant théoriquement sur les travaux de l'École de Francfort (Adorno) et sur ceux de Gramsci. Ce courant a permis de reconsidérer la représentation des classes subalternes dans la culture de masse, et d'examiner les productions culturelles de masse comme autant de véhicules d'idéologies ayant pour effet d'assujettir les classes subalternes. Les travaux de cette école, mariant études littéraires, communications, médias, science, ont été fructueux dans des champs comme les études ethnico-raciales, les études de genre, les études des milieux populaires, et avec eux, il devenait possible d'examiner toute production culturelle de masse comme une construction spécifique de la race et de l'ethnicité, du genre et de la classe, en considérant bien sûr ces catégories comme autant de façons de réifier des groupes. Des cultural studies, deux lecons pouvaient être tirées. D'abord celle de l'étude de la culture comme lieu de constructions plurielles, mettant fin encore une fois à toute élaboration conceptuelle de la culture de type configurationniste, homo-généisant et totalisant. Ensuite, les cultural studies ont pénétré les programmes d'études de genre, d'études ethnicoraciales, d'études gay et lesbiennes, puis les queer $^{5}$, et les disability studies. Les minorités, vraisemblablement, ont trouvé en leur sein, et en périphérie des points de vue dominants de l'anthropologie, des moyens de faire l'étude critique des représentations à propos d'ellesmêmes, et de proposer des conceptualisations alternatives. Le travail sur le langage est au cœur de la majorité de ces recherches. Le regard du centre de la discipline sur ces travaux, est parfois dur, car certains anthropologues mais pas tous, cela est sûr, les considèrent comme le royaume de la rectitude politique (en Amérique) ou du communautarisme (en Europe et surtout en France). Cela n'est pas complètement faux mais ne saurait s'avérer une raison suffisante pour se contenter de rejeter du revers de la main la " parole des autres ", migrant au sein même de la discipline de la marge vers un centre en redéfinition, obligé plus que jamais à cette redéfinition. Ainsi, au sein des black studies ${ }^{6}$ a-ton prêté attention aux constructions scientifiques de la race et du pouvoir, au sein des gay and lesbian studies on a fait de même au sujet du genre et du pouvoir, donnant d'ailleurs lieu à au développement des queer studies, probablement le mouvement le plus radical en ce qui concerne l'étude du genre abordé ici comme chez Judith Butler en tant qu'acte performatif, et enfin, les disability studies ${ }^{7}$ ont-elles ajouté au sein de ces mouvements le débat les relations entre pouvoir, corps différents et handicap. Pour l'anthropologie, il est possible que chacun de ces mouvements puisse être vu comme trop radical, trop enchevêtré dans la découverte de nouvelles subjectivités, noyé dans l'apologie de sa différence, négligeant du coup l'horizon du commun. Il semble sans doute plus juste d'examiner tous ces mouvements comme autant de façons de reconsidérer le projet anthropologique, universaliste. Il faut sans doute se réjouir de "l'avènement de la parole de l'autre " au sein même de la discipline. Il faut prendre au sérieux ce travail de ré-définition des frontières du savant et du profane, du soi et de l'autre, du sujet et de l'objet, que seule l'anthropologie, et sa capacité de réflexivité et d'indiscipline, peut accueillir.

Le courant postcolonial est certes venu renforcer et réaffirmer les critiques des courants antérieurs, avec ceci de particulier que la relation anthropologique concernée, et ses constructions inhérentes, ne touchait point dans ce cas précis les différences (et les inégalités) de genre, de race, de classe, de corps, d'orientation sexuelle, mais bien la relation coloniale elle-même, et ses constructions politico-scientifiques. L'institution coloniale fut, comme on le sait, une machine destructrice, rejoignant la totalité des institutions locales, des cultures locales, et les plis les plus intimes des subjectivités. C'est du côté de l'Asie et du Moyen Orient, et par le biais d'intellectuels érudits originaires d'anciennes colonies et ayant étudié aux États-Unis ou ailleurs dans les pays anglo-saxons, que sont venus les post-colonial studies ${ }^{8}$, lesquelles, tout comme les cultural studies, ont pris le chemin des départements de littérature anglaise avant de faire un saut du côté de l'anthropologie. Ce détour n'est peut-être pas le fruit du hasard, car il semble que l'anthropologie tarde à assumer complètement la critique issue $\mathrm{du}$ postcolonialisme et à prendre acte du tournant que doit accomplir la discipline. L'enjeu n'est probablement pas le même dans les études littéraires et dans les départements d'anglais lorsque les chercheurs s'intéressent aux "contextes sociaux" de la littérature des anciens empires. Les auteurs des textes sur le postcolonialisme publiés dans la revue l'Homme en 2002, étaient tous autres que francophones et plusieurs n'étaient pas anthropologues, ce qui est remarquable. Au sein des postcolonial studies, 
des auteurs comme Said, Bhabba, Spivak ${ }^{9}$, sont des sources intarissables qui ont stimulé la relecture critique des travaux anthropologiques sur l'Inde (via les études subalternes, associant historiens et littéraires). Ils ont favorisé le décloisonnement de l'anthropologie et des études régionales (aera studies), de plus en plus désertées, en suggérant que la "région " ou "l'aire culturelle" résultèrent d'un découpage issu du pouvoir colonial, mais malheureusement repris par les historiens, les sociologues et les anthropologues. Leurs auteurs ont su dépasser le constat des souffrances passées et actuelles produites par le rapport colonial. L'importance de ce courant réside moins dans la répétition dénonciatrice du rapport colonial et de ses conséquences dévastatrices, ce que bien des auteurs avaient affirmé et démontré de diverses manières, mais plutôt dans la recherche difficile d'une posture que résume très bien l'affirmation célèbre de Spivak, traductrice de Derrida, et auteur de nombreux ouvrages théoriques sur le postcolonialisme : Subaltern can't speak. Ce qui signifie en clair : comment parler le langage vernaculaire et universel à la fois ? Comment faire retour sur son langage propre si le rapport colonial produit un tel sujet qu'il ne peut qu'être extérieur à lui-même, parlé par un autre ? Comment en particulier, pour un intellectuel du tiers-monde, est-il possible d'accéder à la posture universaliste, prétendre parler de façon générale, user de la langue de disciplines comme l'anthropologie ou la philosophie, tout en restant local et en prétendant sortir du rapport colonial ? La réponse de Spivak est que cela est une impossibilité, qu'il n'y a pas de langue subalterne, et qu'une voie possible est celle de l'essentialisme stratégique. Ce qui veut dire en certains lieux parler la langue vernaculaire du local et en faire une stratégie identitaire mais ne pas se contenter de cette position et cultiver la langue de l'universel de façon à multiplier les lieux stratégiques de parole et surtout d'élaboration de savoir. Les études postcoloniales ouvrent ainsi la voie à des formes de savoir anthropologique qui pourraient dépasser le grand partage et converger vers une anthropologie symétrique, tout en faisant de certains sujets du savoir anthropologique des auteurs d'un savoir anthropologique inédit. Les travaux de Edward Said sur l'orientalisme entrent tout à fait dans cette voie. Les études postcoloniales pensent véritablement ce que devient (ou ne devient pas) l'anthropologie : l'autre, une fois de plus, n'est point réduit à son altérité exotique, à l'ombre de la raison. Quand Lévi-Strauss affirmait qu'il fallait " s'ouvrir à la raison des autres", signifiant ici la pensée sauvage, avait-il mesuré ce que cela pouvait vraiment impliquer. Que deviendrait l'anthropologie faite par ces mêmes autres désormais ouverts à "notre raison »? Le succès du travail d'Arjun Appadurai n'est probablement pas le fruit du hasard : lui-même issu des études postcoloniales et d'origine indienne, sa réflexion sur les processus de mondialisation, tout en étant l'œuvre d'un brillant intellectuel, est issue d'une expérience du mélange, des réseaux diasporiques qui défient les "genres ethniques », et de la mondialisation vue par le bas.
L'anthropologie à l'ère des études postcoloniales et de l'essentialisme stratégique n'est pas une sinécure. Dans un pays comme le Canada, mais aussi au Brésil, au Mexique et en Australie, les populations indigènes ne se laissent plus étudier sans réclamer la propriété intellectuelle des œuvres ou tout au moins l'usage exclusif. Nombre de thèses se font dans un contexte difficile car les indigènes exigent des contrôles accrus sur ce qui sera dit et ce qui sera divulgué de leur savoir. En certains lieux, comme en Amazonie, il devient de plus en plus difficile d'obtenir les permis de pénétrer les zones où vivent les indigènes. Ils refusent de plus en plus le statut de simple sujet de l'observation anthropologique et de réservoir de savoir; plusieurs d'entre eux parmi les leaders sont devenus historiens, avocats, anthropologues, créateurs, chanteurs, effectuent des alliances pour tirer partie d'un rapport trop longtemps largement inégalitaire. Ils savent que sans essentialisme stratégique, rien ne changera. Le durcissement des identités, s'il n'est pas souhaitable, s'inscrit dans un rapport postcolonial; qu'on le désire ou non, l'anthropologie doit désormais composer avec sa contestation interne par des sujets transformés en auteurs d'œuvres différentes et définisseurs de leurs identités et de leur culture.

Le mouvement des anthropologies périphériques est international, voire transnational, parce qu'il est porté par des forces qui sont elles-mêmes internationales et transnationales. Il est d'abord porté par les mouvements sociaux, dont les frontières sont de plus en plus floues et mouvantes, notamment les mouvements féministes, gays et lesbiens, queer, black, panamérindiens; il est aussi porté par des intellectuels présents sur les scènes des grandes villes du monde et au-delà, aidés il est vrai, par les activistes des droits humains ; ensuite, on ne saurait les oublier, il est enfin porté par les mouvements migratoires et diasporiques, d'où sont issus les étudiants des pays du tiers-monde et les études postcoloniales.

Le mouvement des anthropologies périphériques, parce qu'il contient en lui le germe de formes rigidifiées d'identités, comporte des dangers, dont celui de tuer à la base tout projet de renouvellement du discours anthropologique, parce qu'il pourrait être confiné à la reproduction de ce que l'on connaît déjà trop bien. Celui qui s'autorise le savoir sur lui-même ne saurait être le seul à pouvoir relater son expérience, et si c'était le cas, il enfermerait alors la parole et s'inscrirait dans une téléologie paradoxale. Ce même mouvement contient toutefois d'autres germes, et c'est bien sûr ceux-là qui intéressent, en sortant l'anthropologie et les groupes qu'elle a constitués en objets des anciens dualismes. Cette anthropologie du tiers, combinaison possible de paroles jamais arrêtées sur les expériences du monde et des altérités retrouvées en soi (pour l'anthropologie) et de l'expérience même des altérités (pour tous), et de la rencontre réflexive de ces paroles, a tout le mérite de sortir la discipline des nombreuses impasses où elle se trouve. La recherche dans cette direction pourrait être salutaire, en particulier si elle permet de ne pas fragmenter les mouvements 
intellectuels évoqués en de simples tendances et courants communautaristes, mais plutôt de relier les uns aux autres par leurs contours paradigmatiques, comme nous tentons ici de le faire, ce qui est inhabituel. Il faut aussi rappeler que ces courants émergent au sein de l'anthropologie mais aussi de d'autres disciplines, se logeant au sein de carrefours d'idées et de pratiques émancipatrices. Ce sont donc également des frontières des communautés et des savoirs dont il est question.

\section{Particularismes, universalité et éthique}

L'anthropologie se modifie ainsi par ses frontières géo-culturelles, communautaires et épistémologiques, tout en cherchant le langage le plus adéquat pour nommer ces changements successifs d'échelle. Elle introduit par ces changements d'échelle une forme de connaissance qui prenne en compte les voix singulières des particularismes dans des espaces géo-culturels décloisonnés. La discipline, engagée sur ce chemin, redessine les contours du particulier et de l'universel. Revenons d'abord sur certains de nos propos de départ, et sur certaines des conditions de la globalisation ou de la mondialisation.

Cette dernière est une vieille histoire avec des temps forts plus connus que d'autres comme l'expansion de l'Empire Romain, la christianisation (accompagnant notamment la Conquête des Amériques) ou l'islamisation. La caractéristique de ce que l'on appelle aujourd'hui la mondialisation est la création de nouveaux rapports à un espace qui devient unique (et donc nous rapproche les uns des autres) et à un temps qui s'accélère (et crée de la distance et de l'exclusion). L'espace est amené à se contracter tandis que le temps est conduit à se rétracter. Les distances qui paraissent caractéristiques du premier se trouvent annulées tandis que la temporalité dans laquelle nous évoluons devient étrange, tant le futur nous semble incertain alors que le passé se voit absorbé par un présent permanent, ce qui provoque un certain engourdissement de la sensibilité et des possibilités d'agir.

Le monde d'aujourd'hui tend ainsi à se rapprocher du film que le réalisateur chinois Jia Zhan-Ké a appelé précisément The world (2005) qui met en scène la clôture d'un espace-temps rétréci et concentré. The world, univers d'illusion et de falsification dans lequel il n'y a plus que des produits de synthèse, est le nom donné à un parc d'attraction de Pékin qui reproduit à une échelle réduite les monuments les plus célèbres de la planète. Il permet à ceux qui le parcourent de faire le tour du monde à pied en quelques heures sans quitter la capitale chinoise.

Pour désigner ce processus poussé à son paroxysme dans le film de Jiaz Zhan-Ké, mais qui pourrait bien préfigurer ce qui nous attend, nous disposons de trois termes, globalisation, mondialisation et universalité. Nous allons interroger les deux premiers puis montrer que le troisième, à condition d'être distingué de l'universalisme idéologique, est susceptible d'ouvrir un tout autre horizon d'action et de connaissance.

La mondialisation se réfère au monde, la globalisation au globe et au global. Ce sont deux notions régies par le concept de complétude et de totalité, y compris dans un sens maussien. La globalisation contemporaine est une totalisation, mais c'est une totalisation mercantile dans laquelle la loi économique du marché international est devenue toute puissante et tend à occuper tout l'horizon, la création artistique, la "culture" devenant des valeurs de consommation, des "produits " équivalents à d'autres "produits». Ce processus de domination par privatisation ne crée pas seulement de la monotonie et de l'indifférence (littéralement sans différence), mais de l'exclusion. Il est le fait d'une société devenue sans extériorité, sans dehors (au sens de Blanchot) abandonnée à un capitalisme extrême, sans contrepoids, sans limite.

Cette mondialisation prédatrice, comme au début de la Conquête des Amériques, mais pourvue de moyens techniques dont ne disposaient pas les Consquitadors, est devenue pour un grand nombre de populations une catastrophe, d'abord écologique, mais aussi économique et surtout humaine. C'est ce que nous montre avec force $L e$ cauchemar de Darwin (2004) réalisé par le cinéaste Hubert Sauper et sur lequel nous allons fixer notre attention.

Depuis le début des années 1960 a été introduit dans le lac Victoria un poisson d'une espèce allogène, la perche du Nil, qui a fait disparaître la majorité des autres espèces. Ce nouveau venu, aux très grandes qualités gustatives, est pêché puis préparé par les autochtones dans une dizaine d'usines de la ville de Mwanza située sur les rives du lac. L'intégralité des filets, prêts à être consommés, sont acheminés jusqu'à des avions cargo, d'énormes Iliouchine, qui les transportent en Europe et au Japon. Ce qui reste sur place, ce sont précisément les restes, les arêtes et les têtes jetées dans des décharges nauséabondes dans lesquelles pullulent les asticots et qui vont constituer une partie de l'alimentation de la population. Bref, des bénéfices du développement économique, les Tanzaniens ne se partagent que des déchets. Mais ce n'est pas tout. Les Iliouchines qui décollent deux fois par jour en transportant des centaines de tonnes de filets ${ }^{10}$ n'arrivent pas vide. Ils sont remplis d'armes destinées à entretenir les guérillas locales des pays voisins.

Voici donc, tel que nous le voyons et l'entendons dans ce film d'épouvante, notamment à travers les propos embarrassés des pilotes russes, une région du monde qui a été le berceau de l'humanité et pourrait vivre de ses propres ressources mais qui est devenue l'un des terrains non seulement de prédateurs, mais de destructeurs. Nous nous trouvons en présence d'un cas particulier d'échanges Nord-Sud (des poissons contre des mitraillettes) provoquant non seulement la paupérisation mais la décimation par les armes ainsi que par le Sida propagé par des prostituées qui occupent les pilotes pendant leur séjour à Mwanza. 
L'intérêt du Cauchemar de Darwin, film sans commentaire, sans pathos, sans surlignage, avançant entre des images et des sons par tâtonnement loin de toute logique de démonstration, est de poser de manière très différente qu'on ne l'a fait jusqu'à maintenant la question de la mondialisation. Appréhendée le plus souvent dans les termes d'une confrontation brutale, d'espaces et de temporalités différentes, cette dernière est construite cinématographiquement dans un travail de montage qui est celui du choc et du raccord entre des plans hétérogènes. Ce qui est tout à fait remarquable dans le film de Sauper est d'avoir rompu avec ses habitudes, en particulier dans le long plan séquence du début montrant le reflet bleu d'un avion survolant la surface du lac Victoria.

Dans ce plan là, plus que dans bien des textes savants et a fortiori des enquêtes journalistiques, nous approchons au plus près de la réalité physique de processus qui, dans ce numéro de Parcours Anthropologiques, suscitent nos réflexions. Or, que voyons-nous? Nous voyons qu'il n'y a pas d'un côté un avion, de l'autre un lac, d'un côté la technique menaçante, de l'autre la nature et la sagesse des écosystèmes; d'un côté "l'Occident", de l'autre l'Afrique, mais l'avion et le lac, la technique et la nature, la Russie et l'Afrique ensemble dans le même plan.

Cette logique de la mondialisation comme occidentalisation mercantile provoque des réactions de défense : une altermondialisation avec des projets alternatifs (les mouvements altermondialistes étant un peu l'équivalent de ce qu'étaient les mouvements socialistes dans les premières décennies de la société industrielle), mais aussi une antimondialisation c'est-à-dire un refus global de l'économie capitaliste mais aussi des formes politiques institutionnelles qui paraissent aller de pair avec elle. Pour les populations exclues et spoliées, ce que nous avons eu tendance à considérer comme universel (en France beaucoup plus qu'au Québec) est identifié à ce que l'on appelle au Brésil «le premier monde».

Mais cet universel est une "fausse universalité " pour reprendre l'expression d'Adorno. C'est du global qui est une manière euphémique de désigner le brutal. C'est un universalisme réifiant et destructeur (tant de la nature que d'industries entières et d'emplois) qui ne peut susciter que des réactions d'antiglobalisation et d'anticosmopolitisme.

Les replis identitaires politiques et religieux se nourrissent de cette association capitalismerichesse-Occident-modernité-Juifs et Chrétiens. À une globalisation qui exclut répond un rejet lui aussi global et sans nuance dont l'expression la plus radicale est le terrorisme. Tout est alors rejeté en bloc, globalement. À une globalisation intégrale de la planète par occidentalisation et mercantalisation, peut répondre une antiglobalisation intégriste.

Les processus de globalisation sont donc à la fois des processus d'uniformisation et de différenciation pouvant aller jusqu'à la fragmentation et à la sécession, tant le global est susceptible de réactiver des formes éminemment variables de mondialisation, entraînant des effets extrêmement différents. Certains de ces effets conduisent à un appauvrissement des modes de vie et des imaginaires. Certains produisent une très grande créativité.

Toutes les rues piétonnes du monde aujourd'hui se ressemblent et il est frappant de constater avec quelle rapidité elles se transforment pour se ressembler. Si je parcours la rue de la République à Lyon, je trouve les mêmes commerces, les mêmes magasins, les mêmes restaurants, les mêmes cinémas projetant les mêmes films que dans les cinémas d'une rue piétonne de São Paulo ou de Montréal. Mais si, me dirigeant place Bellecour, je tourne dans la rue de la Barre, je rencontre une salle d'art et d'essai bien connue des Lyonnais où mon imaginaire va être fécondé par ce qui vient de Corée, d'Argentine ou d'Iran. Découvrant les films de Hong-Sang-Sou, de Lucrecia Martel ou de Kiarostami, je m'aperçois que le mastodonte du cinéma commercial réactive l'extrême vitalité des cinémas indépendants qui se renouvellent aujourd'hui à partir des périphéries. Il en va de même des cuisines, à la fois ressemblantes et dissemblantes. Le couscous est devenu l'un des plats préférés des Français. Jamais il n'y a eu autant de restaurants « ethniques » qu'aujourd'hui.

Les mondialisations sont extrêmement différentes selon les situations, les histoires respectives, les mémoires, les imaginaires et bien sûr les forces en présence. Elles conduisent les cultures soit à se dissoudre (comme le montrent les derniers livres d'Yves Michaud ou de Zygmunt Bauman ${ }^{11}$ ), soit à se durcir, soit à se complexifier et à se mêler sans pour autant se confondre. Elles peuvent permettre la reconnaissance internationale de la singularité (notamment des peuples autochtones) et leur appartenance à une commune humanité.

La condition de l'homme et de la femme aujourd'hui étant la possibilité de vivre dans une multiplicité de temps et d'espaces différents, mais aussi de voix singulières ; l'anthropologue se doit alors d'articuler, sur les bases d'une ethnographie la plus rigoureuse possible, plusieurs dimensions : le mondial, le national, le régional, le micro-local. Dans le mouvement de la pensée anthropologique, les changements d'échelle que constituent le décloisonnement de l'espace géo-culturel et l'avènement des groupes minorisés comme sujets auteurs, critiques et théoriciens, obligent au recadrage du champ de la discipline, et à un double décentrement : celui du cadre physique (le terrain dans un nouvel espace-temps) et celui du cadre interprétatif (qui parle désormais, de qui et comment?). Il faut comprendre que le décentrement inaugural de la discipline ne lui suffit plus. Il convient alors, à notre avis, de réinterroger la notion d'universel et d'abord d'univers qui recèle une très grande richesse sémantique. L'univers, en effet, ce n'est pas l'un, c'est l'un tendu vers l'autre, c'est la flexion de l'un, sa courbure. Ce qui nous incite à délier la notion d'un universalisme abstrait, anhistorique, global et général, d'une universalité qui ne peut être «l'Occident" (et 
donc l'occidentalisation) mais ce qui déborde "l'Occident". C'est sans doute pourquoi les anthropologies périphériques parlent à la fois des singularités et de l'accession à une commune humanité auquel tous sont conviés dans sa définition. C'est sans doute aussi pourquoi les mouvements altermondialistes recherchent le local et le valorisent en même temps que leurs stratégies appellent à un global « incluant ».

On s'aperçoit alors que dans cette démarche (qui ne cesse évidemment d'être creusée par un écart entre l'idéal et la réalité) l'universalisme métaculturel dont s'est abreuvée l'anthropologie n'est qu'un communautarisme déguisé. C'est un communautarisme construit à partir des notions qui n'ont rien d'anthropologique mais qui sont véritablement androcentriques et souvent même chromatocentriques. Cet universalisme par capitalisation de signes (homme-blanchétérosexuel-jamais malade-toujours en formepropriétaire ou copropriétaire de tous les biens et de toutes les valeurs) est réfractaire aux situations de vulnérabilité ${ }^{12}$. Il crée de l'exclusion cumulative.

L'universel, à l'inverse, introduit une rupture dans la logique du centre, de la centralité, des capitales et du capital. Il ne peut être toujours et partout identique. Il ne saurait être « l'Occident" parce que ce dernier est sans cesse débordé par un dehors et c'est à ce débordement questionné que doit contribuer une anthropologie moderne. Cet universel ne surplombe pas, ni se supervise, ni n'oriente notre commune humanité. Il est en permanence ouvert au multiple. Ainsi le fait d'être français ou québécois (ou de le devenir) n'a$\mathrm{t}$-il rien d'homogène, car il y a plusieurs manières de l'être ou de le devenir, plusieurs manières de plier la culture française ou la culture québecoise. Celles-ci ne sont «composées » d'«éléments » que si l'on tient à la logique du structuralisme. On peut les comprendre différemment: formées de plis polonais, italiens, arméniens, juifs, arabes, ... (France), de plis amérindiens, anglophones, franco-phones, latinos, ... (Québec).

Pour Gilles Deleuze, le multiple doit être distingué du pluriel. Il «n'est pas seulement ce qui a beaucoup de parties, mais ce qui est plié de beaucoup de façons ${ }^{13}$. Cette multiplicité nous incite à mesurer les limites d'une pensée de la totalité (concept clé d'une conception paresseuse de la globalisation) et à explorer la pertinence d'une pensée de la modalité ${ }^{14}$ c'est-à-dire des modulations et des flexions infimes de nos comportements. Ces derniers sont difficilement compréhensibles dans la seule logique catégorielle des systèmes de relation et d'opposition entre des éléments (ou «traits culturels ») préexistants.

Bref, la notion d'universel vers lequel nous nous orientons et qui est un horizon de connaissance à défricher n'a rien de définitif, d'arrêté. Elle n'est pas donnée mais à construire ensemble. Or l'un des lieux de construction de cet Universel métissé $^{15}$ est certainement l'université, espace résolument cosmopolite de discussion et de réfutation. Cette notion d'universel peut aussi se construire par la multiplication des lieux de débats au sein d'un espace public cosmopolite.

Cette notion, on le voit, s'oppose à l'universalisme essentialisé et réaffirmé une fois pour toutes comme peut l'être l'universalisme qualifié en France de "républicain ». Ce dernier a tendance à concevoir l'universel comme être ou comme état (d'indivision voire d'indifférence). Il a beaucoup de difficultés à envisager l'universel comme devenir c'est-à-dire comme éthique. Or ce dernier, les anthropologues en ont fait l'expérience, n'est nullement toujours, partout et en toute situation identique. Il ne cesse de différer de lui-même.

\section{Francois LAPLANTINE} Francine SAILLANT

Jacques Revel, préface à Giovanni Lévi, Le pouvoir au village, Paris, Gallimard, 1989, p. XXXII.

2 Laëtitia Atlani-Duault, Au bonheur des autres. Anthropologie de l'aide humanitaire, Société d'Éthnologie, Nanterre, 2005.

3 Gamble Sarah, Feminisme and Postmodernism, Cambridge, Icon Books, 1999. DiLeonardo Micaela, Gender at the Cross-Roads of Knowledge, Feminist in the Post-Modern Era, Berkeley, California Press, 1991.

4 During Simon, «Introduction», in The Cultural Studies Reader, London, Routledge, 1993, p. 1-28. Howell Signe, Cultural Studies and Social Anthropology : Contesting or Complementary Discourses ?, in Nugent Stephen et Cris Shore (éd.), Anthropology and Cultural Studies, London, Pluto Press, 1997 : 103-125.

5 Butler J., Le pouvoir des mots. Politique du performatif. Paris, Éditions Amsterdam, 2004.

6 Lancastaer Robert N. (éd.), DiLeonardo Micaela,. The Gender-Sexuality Reader : Culture, History, Political Economy, NewYork, Routledge, 1997. Dirks N. B. et al., Culture/Power/History, A Reader in Contemporary Social Theory. Princetown, Princetown University Press, 1994.

7 Paterson K. et Bill Hugues, "Disability Studies and Phenomenology: the carnal politics of everyday life ", Disability and society, 14, 5, 1999, p. 597-610.

8 Ascroft Bill, Griffiths Gareth, Tiffin Helen, The Post-Colonial Studies Reader, Routledge, Londres, New York, 1995, p.242-243.

9 Spivak G. C., A Critique of Postcolonial Reason. Routledege, NewYork, 1999. Spivak G. C., The Post-Colonial Critique. Routledege. 1990

10 En France, 2267 tonnes de filets de perches du Nil ont approvisionné les rayons de poissonnerie des supermarchés, le double de l'année précédente.

11 Yves Michaud, L’art à l'état gazeux, Paris, Stock, 2003. Zygmunt Bauman, L'amour liquide de la fragilité des liens entre les hommes, Paris, Éd. Le Rouerge/Chambon, 2003.

12 Cf. Francine Saillant, Michèle Clément et Charles Gaucher, Identités, vulnérabilités, communautés, Montréal, Éd. Nota Bene, 2004.

13 Gilles Deleuze, Le pli, Paris, Éd. de Minuit, 1988, p. 5.

14 Françis Laplantine, Le social et le sensible. Introduction à une anthropologie modale, Paris, Ed. Téraèdre, 2005.

15 François Laplantine et Alexis Nouss, Métissages. De Arcimboldo à Zombi, Paris, Pauvert, 2001 